\title{
Techniques of data collection and reduction in systematic field observation
}

\author{
RICHARD E. SYKES \\ University of Minnesota and Minnesota Systems Research, Inc., Minneapolis, Minnesota 55455
}

\begin{abstract}
Two specific instances of data collection under field observation conditions are noted: (1) the sampling of police activities and interactions, and (2) the influence of proximity and similarity on informal group formation. Coding and recording of behaviors are described and the management of data detailed.
\end{abstract}

For the last decade, I have been preoccupied with developing the methodology of quantitative, systematic field observation. This methodology includes creating observational languages for particular investigations, validating these languages, training observers, sampling some universe of naturally occurring human behavior, coding behavior into sequential data and reduction of the data to the computer, scale development, and static and sequential analysis using both statistical and mathematical models.

At a very early point, it became evident that collection of field data required invention of a portable, observer-operated encoding device. In 1967, I received a contract from the Manpower Administration, U.S. Department of Labor, for a project entitled, "A pilot study in observational measurement of behavioral factors associated with increased employability of outof-school Neighborhood Youth Corps enrollees." During the initial phase of the project, a category system was developed for the coding of work group interaction (Sykes, Mohring, Whitney, \& Wallen, Note 1). Application of the category system required rapid coding. The late Fraine Whitney and I developed the idea of a portable device which would include a set of switches for entering data and a means of storage. In addition the system would include a way to automatically convert the stored data to computer tape. With the assistance of engineers, we drew up specifications for the system. The Graduate School of the University of Minnesota awarded $\$ 16,000$ for its design and construction. Invitations for bid were sent out, and Electro/General Corporation of Minnetonka, Minnesota, built the system. Glynne Shifflet, Electro/General Corporation design engineer, did the technical design of the first and subsequent systems. The original system was called Minnesota Interaction Data Coding and

The research described in this article was supported by the United States Public Health Service, NIMH, Center for Studies of Crime and Delinquency, Grant R01-MH17917 and Office of Naval Research, Organizational Effectiveness Research Program, Contract N00014-75-0075.
Reduction System (MIDCARS) (Sykes \& Whitney, 1969).

The first system used a tone of different frequency for each switch. The "chords" which constituted the data were recorded on a cassette in a commercially manufactured portable tape recorder. A set of filters detected the tones on playback and converted the signal onto computer tape. The tape recorders operated far out of their advertised specifications and many problems developed, especially when the operator moved around. A second system was built using frequency shift keying rather than tones. While this second system had many problems, it proved usable in field observation if carefully monitored. (The second version of the system was produced commercially by Electro/General.) I used the second version in a study of police behaviors. The present solid-state i)atamyte II is, I believe, the sixth generation of the system. In its present embodiment, it was completely engineered by Electro/General, but incorporates many recommendations for improvement made by me and iny colleagues as a result of extensive field tests on the prototype in the course of an observational field study on informal group formation in Navy training units (Sykes, Note 2). The original device developed in 1967 was designed for field observations limited to $1 \mathrm{~h}$ in duration. During my most recent Navy study, the prototype was used continuously for up to $6 \mathrm{~h}$, running on battery. The police and Navy studies provided opportunity for development of many techniques to facilitate collection and reduction of data on human behavior in field settings.

\section{THE POLICE PROJECT}

The police-civilian confrontations which were common early in the last decade caught social scientists by surprise. They had not studied police work sufficiently even to know the types of tasks typically performed by police officers, much less understand the dynamics of their relations with civilians. Reiss (1971) had pioneered the technique of structured observation during the summer of 1966 , but had not attempted 
to code the actual process of interaction between police and civilians. My hope was that an analysis of interaction as a stochastic process might lead to understanding how police-civilian encounters escalated into confrontation.

\section{Sampling Police Activities and Interactions}

Police patrol work falls into unusually natural macrounits. Uniformed patrol officers usually work an 8 -h shift. During the $8 \mathrm{~h}$, much less than $2 \mathrm{~h}$ on the average are devoted to specific activities. Minutes and sometimes hours of patrolling intervene. These clear boundaries formed the basis of the code, and made much less problematical unitizing problems such as faced Barker and Wright (1955).

All shifts and all cars assigned to each shift over a 7-day period were enumerated. A random sample of cars stratified by shift and by Friday-Saturday or other days was taken at the beginning of each week. The stratified design permitted oversampling of those times when police were busiest. Observers were then assigned to these "rides" in as random a fashion as the exigencies of human scheduling over a many-month period permitted. Thus, an observer on the Thursday Dog Watch would show up at the precinct station during the roll call which preceded the shift, display his identification, and inform the sergeant that he was to ride on the sampled car. Carrying his Datamyte, he then rode the entire 8 -h shift, accompanying the officers on all calls.

\section{Police-Civilian Interaction Code}

Except for routine traffic monitoring, about $90 \%$ of the work of uniformed patrol officers is in response to citizen complaints. The complainant phones the police department and the radio dispatcher directs a particular car to the address given, informing the officers of the nature of the complaint. "Car 220. Take a report. Burglary. 2715 Emerson South. Upstairs." Preliminary field work disclosed that officers used an argot to describe the great majority of their activities (some jurisdictions used statute numbers) and that these could be developed into a category system (see Wallen \& Sykes, Note 3, for a full description of the code).

Work by Stinchcombe (1963) had shown that one important factor in police activity is location, that is, whether it occurs in a public or a private place. The place code designated the type of location.

Since I was also interested in the phase structure (Bales \& Stroatbeck, 1951) of the encounter, it was also necessary to code whether conflict between civilians was occurring at the time the officers arrived. Control statements were hypothesized to be more likely to occur in the first phase of conflict situations.

The numeric code which expressed the observational language was divided into subsets. Each subset consisted of a unique series of numbers, often within a limited range. Examples of these subsets are: the activity code,
$456 \mathrm{nnn}$ or $123 \mathrm{nnn}(1 \leqslant \mathrm{n} \leqslant 9)$; the place code, $7 \mathrm{nn}$ $(n \leqslant 5)$; the state code, $7 n \quad(n \geqslant 6)$; the close code, $4560 \mathrm{n}$ or $1230 \mathrm{n}$; and the outcome code, $963 \mathrm{n}$.

The digits within each subset were ordered so that not only the number itself, but also its location was important. For instance, 45618 was different from 45681. The code was designed so that no number could have more than one meaning. After an element from a particular subset was encoded on the Datamyte, the ENTER (E) switch was depressed, utilizing the option that time was automatically registered each time $\mathrm{E}$ was used. Thus, the series

\section{E time \\ $711 \mathrm{E}$ time \\ $76 \mathrm{E}$ time}

is decomposed in the following manner:

\section{6 (radio dispatch) 1 (crime against property) 8 (take a report) \\ 71 (private space within multiple dwelling) 1 (police invited in) \\ 76 (no overt verbal or physical conflict between civilians at time of arrival)}

The microunit of interaction for coding was the "utterance," all one actor spoke without significant pause or interruption. It proved impractical to use the simple sentence as the microunit in the simultaneous coding of interactions under field conditions.

Several innovations were made in interaction coding including multiple dimensional coding and the use of code abbreviations. Police-civilian interaction has two important dimensions. One is the content dimension as it related to the original complaint or problem. Thus, officers called to take a report about a burglary examine the scene and discuss the burglary with the civilians. But work by Piliavin and Briar (1964), Reiss (1971), and Westley (1953) emphasized the import not only of what officers and civilians said, but how they said it. Police expect civilians to display respect and vice versa. While content may be conceived to be a discrete dimension, respect is continuous. Every utterance, regardless of content, is more or less respectful. Thus, every utterance was coded by role of person speaking, role of person spoken to, content, and respect.

A second innovation was to minimize coding by using abbreviations for the most frequent code combinations. For instance, in the domain of policecivilian encounters, police usually talk to civilians and civilians to police more than police talk to police or civilians to civilians. Therefore, designating $0=$ role ascription for police, and $1=$ role ascription for otherwise undifferentiated civilian, rather than requiring observers to code 01 (police to civilian), 0 used alone 
implied 01 and 1 used alone implied 10 (civilian to police). Full pairs were used only for the infrequent events: 11 (civilian to civilian) or 00 (officer to officer). Then an utterance might be coded as follows:

\section{$0365 \mathrm{E}$}

0 (officer to civilian) 3 (content domain related to the burglary-referring to initial activity code) 6 (a question) 5 (normally respectful--the norm is established during training)

Preliminary work had shown that once a communication enters a particular content domain it tends to remain there. Further, certain grammatical forms usually are complemented by other forms. Questions are usually followed by answers, and these answers usually relate to the content of the question. Accusa- tions are usually followed by admissions or denials and these usually relate to the content of the accusation. An order is usually followed by obedience or disobedience, and this is usually related to the content of the order. This permits certain abbreviations to be used to lighten the coding burden on the observer. Thus, a full response to $0365 \mathrm{E}$ might be:

\section{$1345 \mathrm{E}$}

1 (civilian to officer) 3 (content domain related to the burglary) 4 (answer to previous question) 5 (normally respectful)

This was abbreviated as $19 \mathrm{E}, 9$ implying 345 . Utterances in series may thus be represented as follows: 0365E; $19 \mathrm{E} ; 09 \mathrm{E} ; 19 \mathrm{E} ; 09 \mathrm{E} ; 19 \mathrm{E}$. This is a series of questions and answers pertaining to the burglary, all displaying

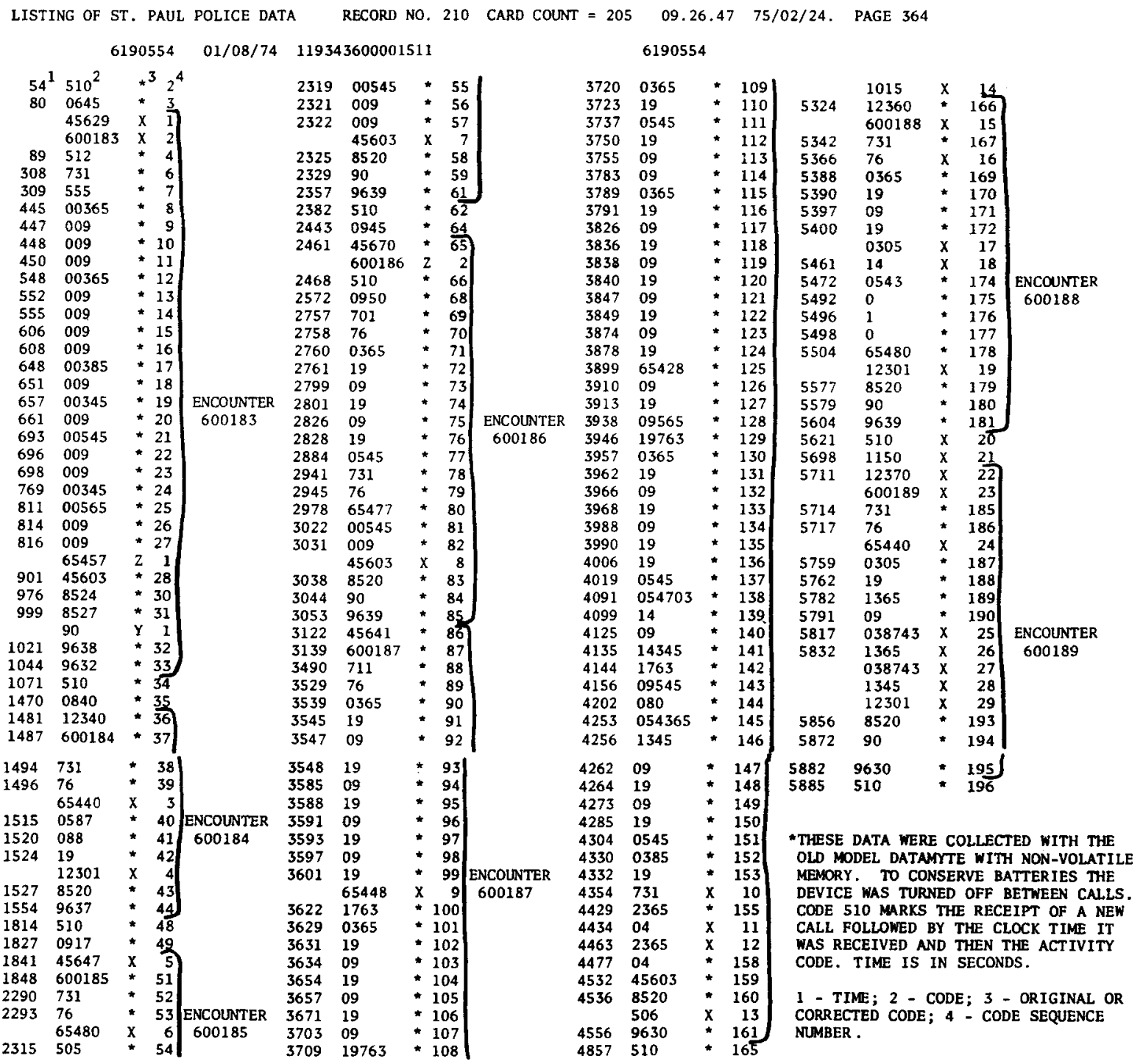

Figure 1. An example of a coded police shift.* 


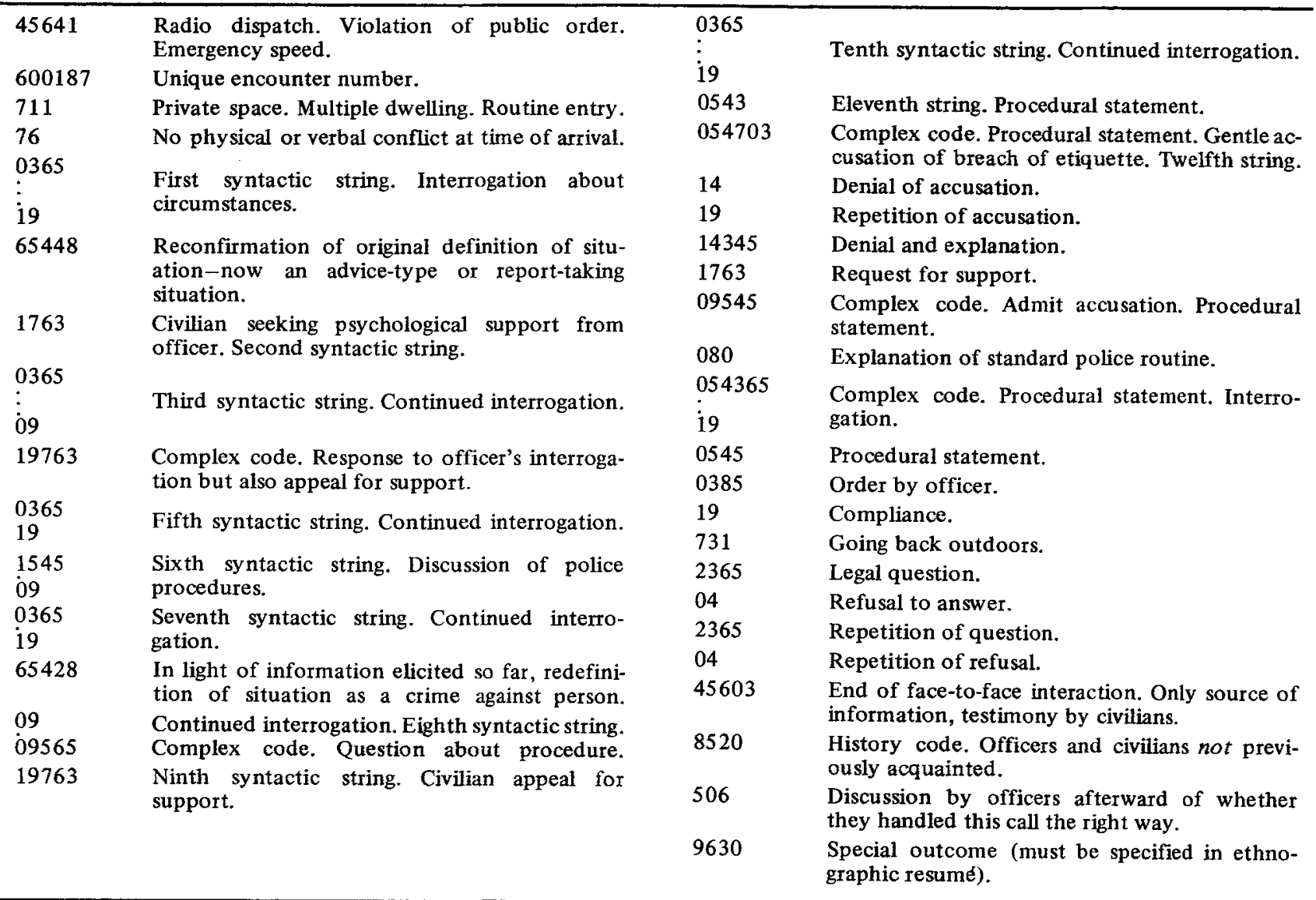

Figure 2. Schematic of an encounter from Figure 1. In addition to the code data, the observer fills out an extensive schedule and writes a brief summary after each encounter. Each printout is checked and corrected by two persons (including the observer).

normal respect. It is termed a "syntactic string." When computer analyzed, the "header code," the 0365, is noted, and all "operators," the 09s and 19s, are converted back to full code. This syntactic string becomes: 0365E; 1345E; 0365E; 1345E; 0365E; 1345E. Most police-civilian interaction is quite routine. It is probably simpler than that of Bales' students solving chess problems.

Police-civilian episodes likewise possess clear-cut endings. The officers depart and go on their way, although sometimes this is from the jail, where they have deposited a prisoner. An appropriate end marker can be entered at the time of departure, permitting calculation of the durations of the episode and its subphases. The close code, $4560 \mathrm{nE}$ or $1230 \mathrm{nE}(\mathrm{n} \leqslant 3)$ is entered. Modifier (the $n$ ) indicates the kind of information made available to the officer during the encounter.

A printout from a relatively inactive shift is displayed in Figure 1. It is not possible to describe the entire code, Police IV, or the data-handling codes which we have used for file management. There are, however, three general principles which code developers should remember: ease of entry, redundancy, and practice.

Ease of entry means that the investigator should design codes that are convenient for an observer to enter without looking at the keyboard. The most convenient combinations are those that go horizontally or vertically across the keys; $123,456,789$ are the simplest horizontal triples; 741,852 , and 963 are the simplest vertical triples. It is much easier to enter 456 and 963 than (say) 916, especially when walking and keeping a subject in visual and audio contact.

It is also advisable to build redundancy into codes. Observers have "slips of fingers." Unique combinations of numbers such as 456 can be entered rapidly and yet do not occur randomly; 456 can be rescued from 46 , 465,54 , or 645 . If a single digit is used as a code, there is no intrinsic way to either recognize or retrieve an error.

Practice is another requirement. Apart from thorough familiarity with the correct operation of the device, full effectiveness depends on recognizing that a key entry recording device is not unlike a calculator, typewriter, teletype, keypunch, or piano. Rapid and accurate entry of codes by a human operator requires practice and good finger dexterity. Operators should practice daily with the keyboard, preferably entering the numeric codes which will be used in the actual research, beginning slowly and working up to rapid speed. Police observer training took 3 months. Because 
the Navy code was simpler, much less time was required. With practice, an observer gradually acquires the facility to enter codes accurately and quickly without having to maintain eye contact with the keyboard.

Conceptually, when a key entry device such as this is utilized, one may distinguish between "accuracy" and "reliability." Accuracy refers to the correct entry of the code that the operator intends to register. Interobserver reliability involves agreement between two or more observers on the code appropriate to categorize a particular event. When a machine such as this is used without practice, inaccuracy often shows up as unreliability. In a series of controlled experiments, as speed of coding increased, inaccuracy increased to as much as $50 \%$ on four-digit codes, even when reliability was perfect.

\section{THE NAVY STUDY}

The focus of the Navy study was the influence of proximity and similarity on informal group formation. I wished to study the emergence of informal groups in a natural setting, and to record the frequency of interaction of individuals as these smaller informal groups differentiated within a larger one. Emergent patterns were to be documented with interaction counts. One way to do this is to observe subjects in newly formed training units, varying in size from 30 to 60 men, interacting spontaneously and simultaneously in the compartment (barracks wing) in which they live.

Because of their number, the physical location, and the decentralized communication pattern, it was not possible to observe all of the men simultaneously. It was necessary to develop a sampling design based on a knowledge of the size of the sample necessary for analysis and inference, as estimated by Larntz (in Sykes, Note 2).

\section{Sampling the Compartment}

In developing the design, consideration was given to several alternatives. In a previous study (Sykes, Larntz, $\&$ Fox, 1976), 6 subjects in a group of 80 had been sampled on an ordered rotation basis. Using a list of the six, the observer physically located the first subject on the list and recorded his communicative behavior; then the second subject was located, and so on. When the sixth subject was recorded, the observer started again at the beginning of the list. This method entailed successive search within a geographical area containing several rooms, one of which was about $160 \mathrm{ft}$ long and contained many people. Finding six subjects was very time consuming. Successively locating an entire unit would have been even more time consuming and would have limited the total number of samples. So any method involving search for individuals was impractical.

The alternative was a form of area sampling. The compartment and lounge were divided into 10 sampling areas. Each area was delimited, based on pretesting, to a geographical area that could normally be scann, quickly by an observer to determine: (1) who was in the area; (2) whether interaction was occurring; and (3) if interaction was occurring, with whom.

After the areas were delimited, random lists (without replacement) of the 10 areas were generated. After pretesting it was found that it was possible to sample all 10 areas in $15 \mathrm{~min}$. The observers were instructed to sample the 10 areas in random order for $15 \mathrm{~min}$. For the succeeding $15 \mathrm{~min}$, they sampled the 10 areas using a different random order. Sampling was carried out at least $6 \mathrm{~h}$ every day for approximately 14 days for each unit.

It was necessary to enunciate several conventions for observers to follow in utilizing this design. The first pertained to subjects lying in the middle of their racks, and therefore in the middle of the imaginary line dividing some sampling areas. Such a person was always considered to be in the area to the rear of the dividing line.

The second convention pertained to the interarea interaction. For obvious reasons, it was necessary to avoid counting a subject twice if, for instance, two subjects were conversing across the boundary of an area. Since both speaker and listener were coded, it would have been possible to code both present in each area, thus compromising the independence of each count. To avoid this, interarea interaction was coded only if the other subject was to the rear of the area being coded. Thus, if Subject $A$ in Area 2 talked to Subject B in Area 3 during the time that Area 2 was sampled, the interaction would be toward the rear and therefore coded. When Area 3 was sampled, since the same dyad would be interacting toward the front, it was not counted.

Finally, by convention (or definition), I utilized a somewhat broader definition of "interaction" than many have used. Often "interaction" is limited to focused verbal behavior. There are several other essentially symbolic activities which are focused, but which may not involve continuous overt verbal behavior. In the first place, interacting includes not just talking, but active listening. Since the observer scanned an area, taking a fix on its occupants, he did not listen to a sequence of turn-taking in conversation. The observation of each area was somewhat similar to a snapshot, recording interaction as of that moment. Listening was considered as important as speaking. The first code indicated the speaker. The subsequent code specified the active listener(s).

Second, mutually focused symbolic activities in which two or more subjects engaged, such as playing cards or craps, were considered interactions. Purely audience-type activities were not considered interaction. To qualify as interaction, there had to be mutual participation in some common focus of interest which involved active participation by the subjects. Since in 


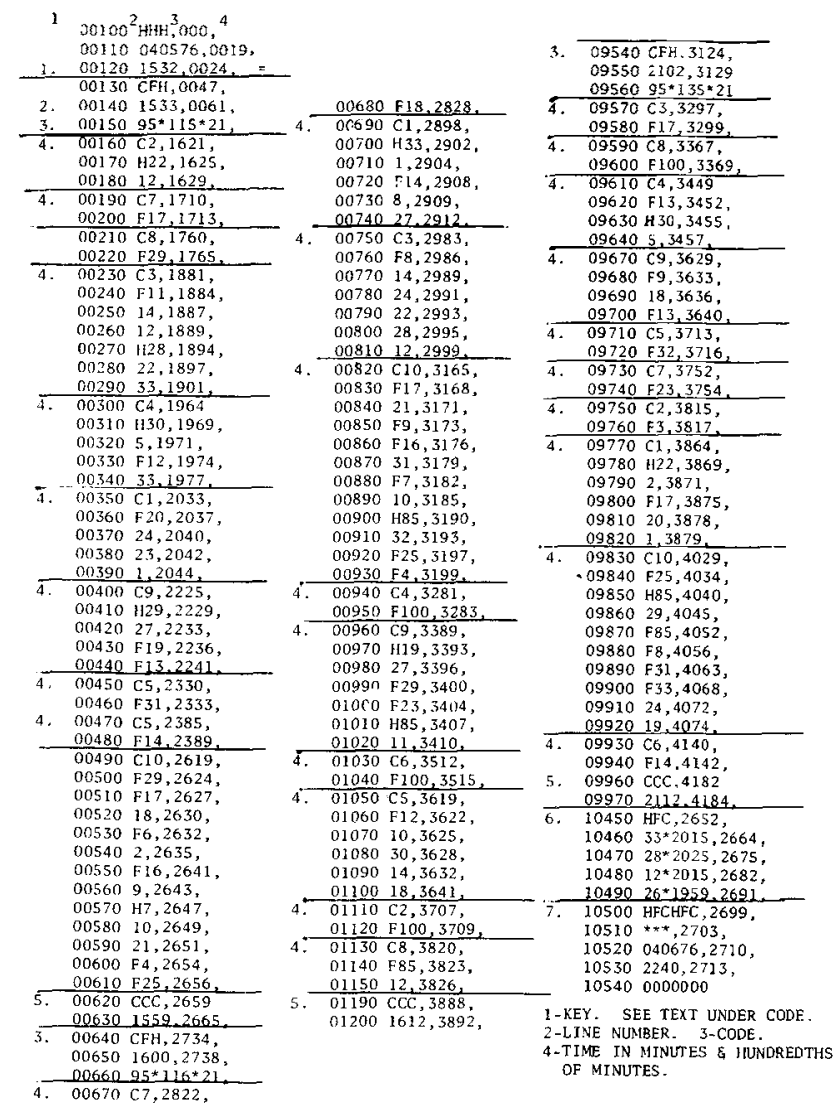

Figure 3. An example of selected sections of a data printout for three sampling periods in the compartment. this context the unit was not a work group, it enabled observers to code important forms of leisure interaction not limited to overt verbal behavior.

In Figure 3 is a printout of three sampling periods from one observation. Figure 4 is the matrix of counts from a full observation. The cells on the diagonal indicate the number of times the subject was coded as physically present in the compartment in some sampling area but not interacting. The off-diagonal cells indicate the interaction counts with other subjects. Only the upper right triangular matrix is necessary, because speaking is counted as equivalent to listening. The count is symmetric. The column above the diagonal must be added to the row for each subject. Marginals may be unequal for several reasons: normal sample variation, observer error, or the subject being absent from the compartment. The toilet, shower, and drying room areas were off-limits to observers. Subjects might also be on liberty during at least part of the period of observation or, occasionally, somewhere else in the barracks. For the statistic appropriate for analysis of such data, see Larntz and Weisberg (1976).

\section{Mechanics of Data Collection}

This design proved practical for the Navy project. Subjects were contacted when they reported for training. The project was explained and informed consent obtained. If an individual consented to be part of the study, I sought permission to take a Polaroid snapshot. The photo was labeled with the subject's project identification number and name. This served as a learning tool for observers, who also spent from

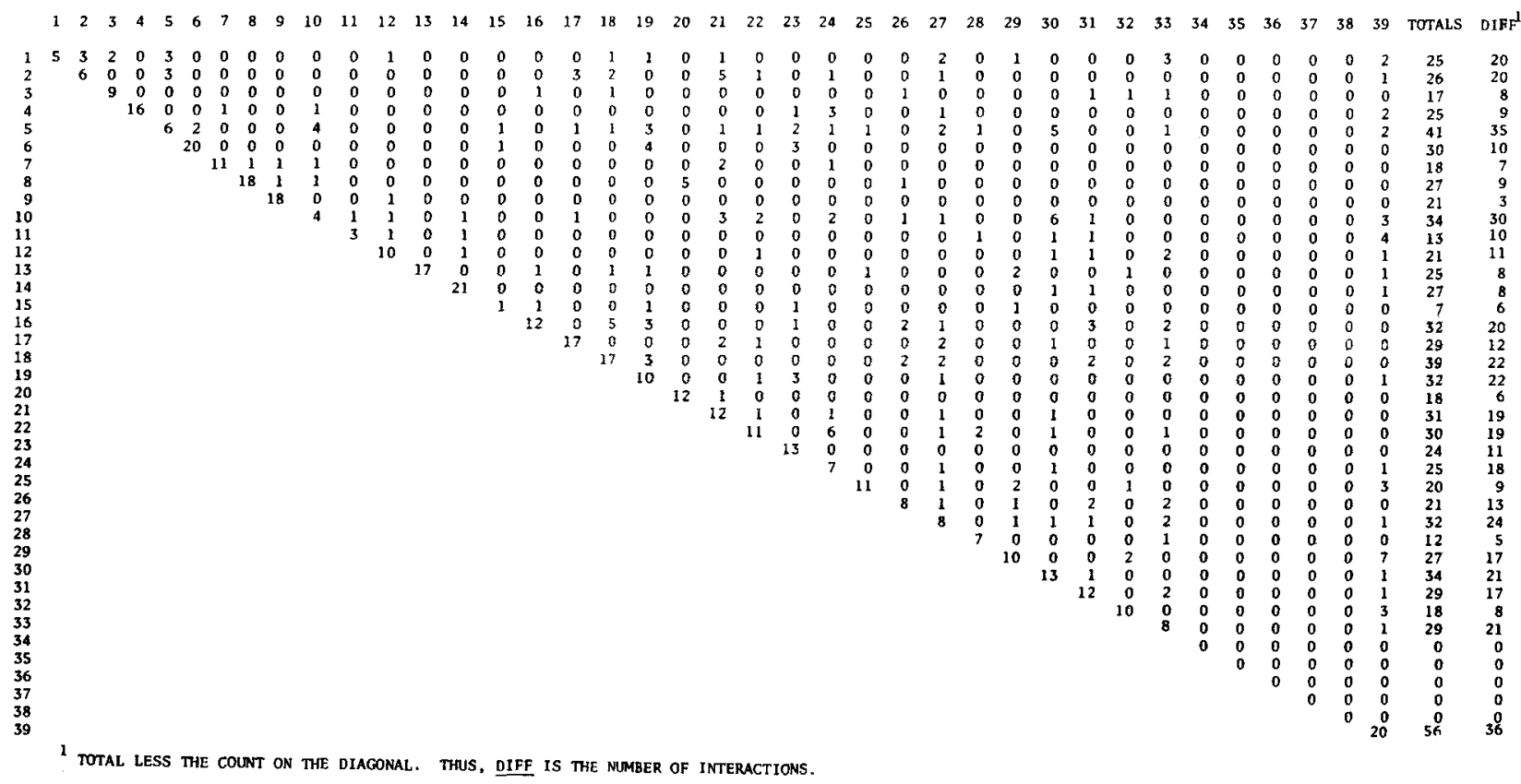

Figure 4. A matrix of counts from a full observation. 


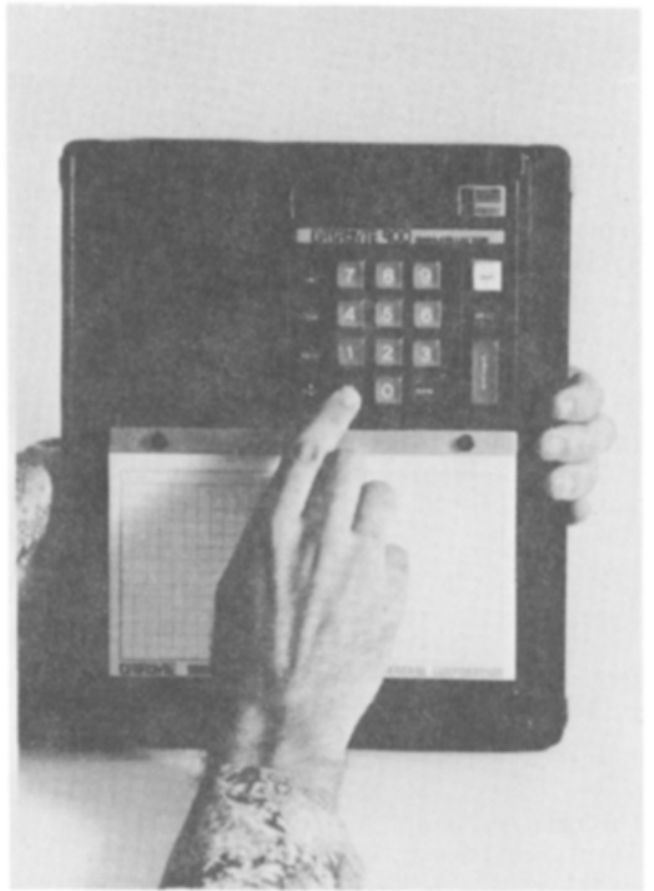

Figure 5. The Datamyte keyboard.

1 to 3 days with the subjects before the unit was officially formed and training began. In this way, the 30 to 50 subjects were individually known to the observers by number and name before the formal data collection began. Observers had at least 2 weeks of concentrated keyboard practice before collecting real (as opposed to practice) data.

During the approximately 14 days each unit was observed, data were recorded by both machine and pencil-and-paper techniques. A prototype, solid-state Datamyte II (see Figures 5 and 6) was used during most of the study. At the end of the daily observation period, the memory was emptied directly onto magnetic tape in a computer 400 miles away through an Execuport portable Teletype terminal. The data were cleaned on-line. The paper-and-pencil data were a back-up in case of failure of the Datamyte II. Figure 3 is an example, not only of the observer code, but also of Datamyte II output.

\section{The Code}

To record data, observers used an alphanumeric observational language which was recorded onto the memory of the Datamyte II through its keyboard. Various combinations of letters and numbers were the vocabulary of the observational language. Each "word" in the language was constrained by certain ordering rules, somewhat like a grammar, as well as by other conventions (see Table 1).

The key in Figure 3 should permit the reader to translate the codes used in that observation. Thus, 3 refers to the third section of the code outline in Table 1: on*sn* $1 \mathrm{n}$; observer number, sample number, and location number.

\section{DATA MANAGEMENT}

The types of data requiring management normally consist of a coded record of behaviors, one or more schedules filled out by the observer, and, more often than not, narrative comments of the observer about special problems experienced during the observation period. Coordination of these different forms of data in a study involving hundreds or thousands of cases is no small task.

Coded protocols are different from written protocols and require different management. In the first place, a coded protocol such as that in Figure 1 is much more complex than the average protocol. While the code has a required grammar, no two cases are alike. Unlike a coded questionnaire, a coded encounter has no fixed format.

Second, each code within a case may be quite complex. Some are many digits long, and only certain numbers are possible within particular digit positions. Thus, in a normal header code in Figure 1, the fourth digit of a four-digit interaction code may only be a 3,5 , or 7 .

Third, behavior of human beings in natural settings is often subject to contingencies which are apt to turn

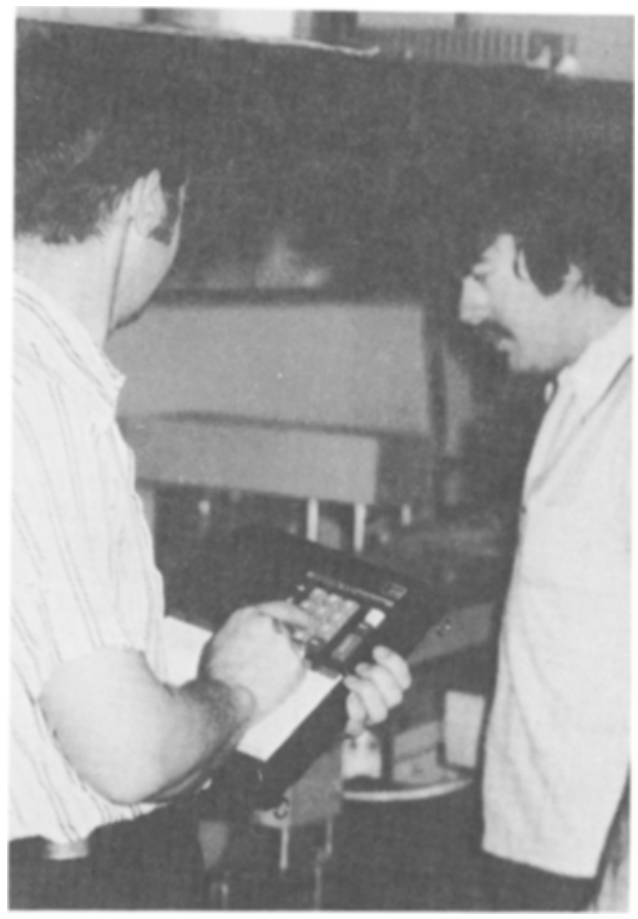

Figure 6. Datamyte in use. It is lightweight and easily grasped with one hand. 
Table 1

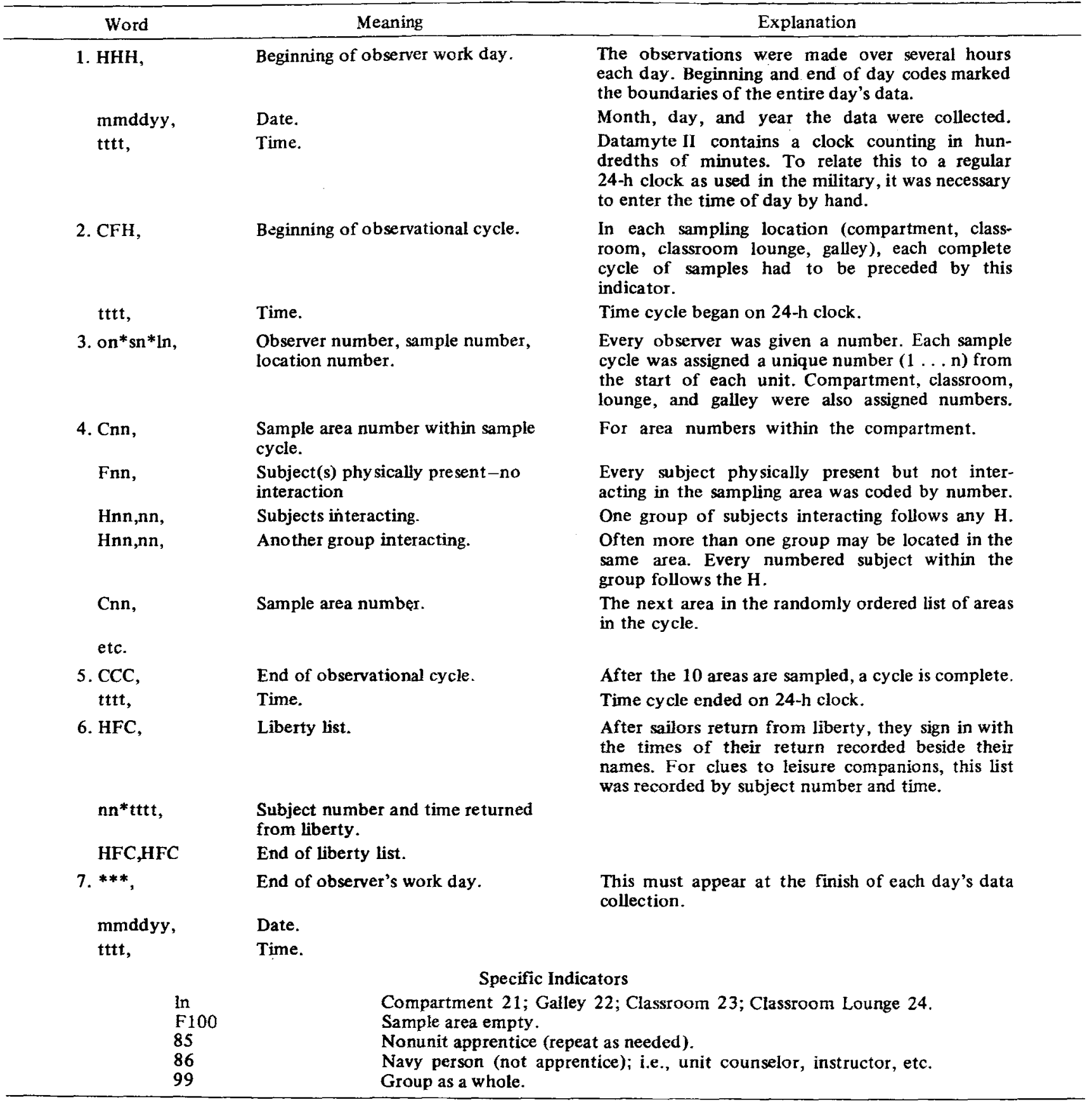

the serious investigator prematurely gray. For example, police officers may be interrupted in the midst of a routine call by an emergency. They handle the emergency and then drive back to finish the routine call. In a coded record, the computer must be able to recognize such an interruption so that eventually the two separated parts of the interrupted case may be combined.

Fourth, because the data from the coded record go directly into computer memory, "cleaning" and editing must take place afterward, not before as with punched cards.

All of these conditions require that very careful written records of the data be maintained and that certain codes be developed which are routinely used by the observer for later computer file management purposes.

\section{Written Records}

It is necessary to maintain an observation record, 
an edit record, an equipment record, and a data inventory. The observation record is the master list of all observations that have been made. The data preserved in this record come from the identification information (IDs) the observer enters in the Datamyte. These IDs contain the basic information of who observed, what was observed, when it was observed, and what equipment was used. This information allows the computerized data to be checked at a later time.

The edit record keeps track of the progress of the data from entry into computer memory to final form. Because of the number of steps involved and the quantity of data, this record is critical to assure that all data are properly prepared for analysis.

Minimization of data loss also requires an equipment record and an "inventory" of the data. The equipment record keeps track of the use, testing, and maintenance and repair of the equipment. Use is recorded on the basis of the IDs. For each use, any operational machine problems are noted. Since each component of the field equipment is constantly rotated with respect to other components, ready identification of equipment problems is possible. If problems exist, testing and repair are undertaken and recorded. The equipment record thus provides the life history of any piece of equipment.

As a further precaution against data loss, an "inventory" is kept of all the pieces of data that should exist for any observation. This assures that no data are lost during the data collection phase either by the observer or by data management personnel. The stages in the process of managing the police data are listed in Figure 7.

\section{Internal Markers: The Bookkeeping Codes}

Both investigator and computer must be aware of unusual contingencies experienced during observation, as well as the meaning of certain unique codes (such as a handentered time code) which otherwise would be meaningless. Bookkeeping codes instruct the computer as to the organization of the data.

For example, in Figure 1, the first code, 510, is an instruction to the computer that the code which follows, 0645 , is a time code. The code 512 , occurring at code sequence number 4 , indicates that this is a previously completed encounter that has started again; 555, occurring at code sequence number 7 , is the equivalent to a footnote. When an event occurs which requires special explanation, the observer enters 555 and narrates the circumstances on his schedule.

Every investigator must be sufficiently familiar with contingencies that influence his research, and with the structures that his data will assume in final form, to anticipate the necessary bookkeeping codes.

\section{PROGRAMMING}

Utilization of this methodology requires five different programming functions: storage; editing; data counts
1. Car-shift sample drawn.

2. Observer assigned to observation.

3. Equipment assigned to each observation.

4. Encounter log sheets numbered.

5. Observation completed.

6. Observer turns in equipment, tapes, and observation log.

7. Observation recorded on observation record with notes from observer.

8. Observation recorded on equipment record with notes from observer.

9. Observation recorded on master list with the number of cassette sides.

10. Tapes translated and logsheet filled out by operator.

11. Data transferred to data file and listed.

12. Are all printouts received? If no, change control deck to skip files received and resubmit.

13. Rip apart printout saving dayfile and tape cataloguetransfer any notes from operator's log to the appropriate cassette printout.

14. Check on edit record as received and record any notes.

15. Check cassettes' IDs.

16. Record cassette side IDs on master list and whether they need to be changed or inserted, and if retained or omitted.

17. If IDs incorrect keypunch ID corrections and submit to computer.

18. Check as received and note condition on equipment record.

19. Equipment $\mathrm{OK}$ as shown by printout? If not, check equipment.

20. If equipment not OK, repair or remove.

21. Record results of any equipment tests on equipment record.

22. Save or erase cassettes-record on observation record.

23. File translation log sheets.

24. Send printouts to observers to edit.

25. Printout returned with encounter logs (observers' schedules) and proofread-record on edit record.

26. If satisfactory corrections not made, return to observer.

27. Record exact observations to be retained on master list.

28. Check observer's corrections.

29. Send edit forms to keypunch-record on edit record.

30. Receive punched and verified cards and edit forms from keypunch-record on edit record.

31. Submit correction cards to edit program-record on edit record.

32. Receive edited printouts-record on edit record.

33. Any error messages? If yes, correct error.

34. Review printouts for additional corrections.

35. Keypunch correction cards and record on edit record.

36. Record as final form on edit record.

37. Finish.

Figure 7. Steps in data collection, reduction, and monitoring during the police project (using older version of equipment with a tape recorder).

by unit and storage of these counts; static data analysis; process data analysis.

The storage program must receive the data from the Datamyte, put it permanently on tape or disk, give each code a sequence number, and provide a printout.

Since no coded record is ever perfect, an editing program is necessary. This is necessary not only to correct or delete incorrect codes, but to reorder codes or series of codes that are correct, but which were entered in an unconventional order or were interrupted. 
In my research, the unit of analysis has seldom been the individual interaction code, but some larger unit, for instance, an entire police-civilian encounter. Normally, a special program is necessary which will scan and unitize the raw data, convert code abbreviations to full codes, count frequencies per unit, and store these frequencies in a form that can be accessed by standard canned programs.

The analysis of the police data required that each complex code be taken apart and separate counts made of its constituent codes by case. Each count locates each case on a particular scale. One encounter can then be examined in terms of several dimensions, for instance, police-civilian respect and disrespect; extent of intracivilian interaction; frequency of orders or questions; and whether a Miranda warning was given the arrestee. These counts are different, taking absolute frequencies per unit for static analysis or transition frequencies of different orders for process or pattern analysis (Brent \& Sykes, Note 4).

Initially, it was my intention to have a program written which would automate the data cleaning and editing task, at least to the extent of recognizing illegal codes and starring them. After much sad experience, I have concluded that this is impractical (the aspiration cost three programmers and a delay of 1 or 2 years). The basic reason for the impracticality is that there are so many contingencies in this type of data that no program can anticipate them. This is especially true when not only a code, but an ordering of codes is required, and when, at the counting stage, everything must be perfect. One or preferably two "editors" can clean and edit the data more quickly, cheaply, and accurately, and enter the changes through the editing program.

I have also found that mere competence and general experience are inadequate qualifications for a programmer. The most productive programmers appear to have (1) a background in statistics; (2) extensive experience with large amounts of messy social scientific data; (3) personalities that derive satisfaction from writing very specific programs which accomplish only a few objectives at a time; and (4) a healthy recognition of the practical limitations of computers, and of those problems which a human can handle more quickly and economically.

Once the counts are made on the raw data, it is less expensive to store them in a form compatible with canned programs than to recount them for each specific analysis. I utilize SPSS for static analysis. The shortage of canned programs for process and pattern analysis has meant that I have had to develop these myself. Readers interested in particular programs which fulfill these five functions may wish to write the author for further information, or for references to a particular programmer. The programs which fulfill these functions are relatively general, except for Function 3 (data counts by unit and storage of these counts), which tends to require programs written for particular coding systems.

\section{CONCLUDING COMMENTS}

Over the past 10 years, I have encountered many difficulties in the use of portable equipment. Almost all of these problems were due either to human error or battery problems. The current model, Datamyte, was designed to be as "human proof" as possible. The battery is improved, but users must still keep careful records of battery use and follow instructions for their charging, recharging, testing, and care. Batteries are eccentric, so careful records should be kept of individual battery use in order to prevent or trace failures. This is especially important if a large project using several encoders and many batteries is being conducted. Human operators must be carefully trained in proper operation of the device, and practice using the keyboard is necessary. Even with practice, an observer with poor finger dexterity will continue to make mistakes.

Use of Datamyte requires considerable investment, not only in the device, but in observer training and in programming, as well as an extensive effort in the development of a code. Therefore, I would not advise a potential user to invest in a machine casually; use for basic research requires a major commitment.

\section{REFERENCE NOTES}

1. Sykes, R. E., Mohring, P., Whitney, F., \& Wallen. D. A pilot study in observational measurement of behavioral factors associated with increased employability of out-of-school Neighborhood Youth Corps enrollees. Minneapolis: University of Minnesota, 1969. Pp. 291. (Mimeo)

2. Sykes, R. E. A design for observer sampling of member interaction in a large, spontaneous group (Tech Report 3). Minneapolis: Minnesota Systems Research, 1977. (Mimeo)

3. Wallen, D., \& Sykes, R. E. Police IV: A code for the study of police-civilian interaction. Observations 6. Minneapolis: Minnesota Systems Research, 1974. Pp. 318. (Mimeo)

4. Brent, E. E., \& Sykes, R. E. The interactional bases of police-civilian confrontation: A Markov process model of social interaction. Manuscript submitted for publication, 1977.

\section{REFERENCES}

Bales, R. F., \& Strodtbeck, F. L. Phases in group problem solving. Journal of Abnormal and Social Psychology, 1951. 46. $485-495$.

BARKER, R. G.. \& WRIGHT, H. G. Midwest and its children. New York: Harper \& Row, 1955.

LARNTZ, K.. \& WEISBERG, S. Multiplicative models for dyad formation. Joumal of the American Statistical Association, 1976, 71, 455-461.

Piliavin, l., \& Briar, S. Police encounters with juveniles. American Journal of Sociology, 1964. 70, 206-214. 
ReISs, A. J., JR. The police and the public. New Haven: Yale University Press, 1971.

StinchCombe, A. L. Institutions of privacy in the determination of police administrative practice. American Joumal of Sociology, 1963, 69, 150-160.

Sykes, R. E., Larntz, K., \& Fox, J. C. Proximity and similarity effects in a class of naval recruits. Sociometry. 1976, 39. 263-269.
Sykes, R. E., \& Whitney, F. Systematic observation utilizing the Minnesota interaction data coding and reduction system. Behavioral Science, 1969, 14, 167-169.

Westley, W. A. Violence and the police. American Journal of Sociology, 1953, 59, 34-41.

(Received for publication July 5, 1977; revision accepted August 1, 1977.) 